\title{
WAVE AND PARTICLE MEASUREMENTS NEAR THE LOWER-HYBRID FREQUENCY*
}

\author{
P. L. Colestock and W. D. Getty \\ Electron Physics Laboratory, Department of Electrical and Computer Engineering, \\ The University of Michigan, Ann Arbor, MI 48109, U.S.A.
}

(Received 22 August 1976)

\begin{abstract}
Experimental results are presented on lower-hybrid waves excited in a plasma column by an electrostatic coupler. It is found that the observed wave patterns, which clearly show resonance cones, are as predicted by a theory based on the superposition of bounded-plasma eigenmodes for a source with a wide $k_{z}$ spectrum, and are also consistent with the WKB resonance-cone formulation. Results on electron heating and plasma loss produced by lower-hybrid waves are also presented.
\end{abstract}

\section{INTRODUCTION}

RECENT theoretical and experimental studies by SimonutTI and PARKER (1974), Bellan and Porkolab (1974) and Colestock and Getty $(1974,1976)$ indicate that lower-hybrid waves excited by finite-sized electrostatic sources give rise to resonance cones in the plasma response, and that these resonance cones can be viewed as a superposition of bounded plasma eigenmodes. The resonance cones follow curved trajectories in an inhomogeneous plasma which become asymptotic to the lower-hybrid resonance layer at large axial distances from the source. For finite-length cylindrical sources concentric with the plasma, the resonance cones emanate from the ends of the source and may reflect at the column axis provided no resonance layer occurs in the plasma.

Resonance cones near the lower-hybrid frequency were first observed by BRIGgS and PARKer (1972). A cylindrical, axially-localized source was used and a single set of resonance cones was excited. Their results agreed qualitatively with a planar WKB theory but no attempt was made to quantitatively verify the theory.

HOOKE and BERNABEI (1972) later reported measurements of lower-hybrid waves excited by long spilt-cylinder plates. A radially localized maximum in the $R F$ potential accompanied by a phase shift was observed over a range of frequencies near the lower-hybrid frequency. The maximum occurred at densities where the condition $\omega \geqq \omega_{L H} \simeq \omega_{p i}$ was satisfied. The deviation from equality in this condition could not be explained on the basis of plane-wave theory alone. Since their measurements were made at a single axial position, resonance cones in the plasma were not readily apparent in their results. Similar measurements have been reported by RaIMbaUtT and SHoHet (1975) and Moresco (1973). Measurements of single lower-hybrid waves with a narrow $k_{z}$ spectrum have been reported by Bellan and Porkolab (1975) and Stenzel and Gerelman (1975).

In this paper experimental measurements of the plasma response are presented for finite-length split-cylinder excitation that is similar to that used in the Hooke and Bernabei experiment. The purpose of the measurements is to verify the theoretical predictions for excitation of resonance cones and other wave phenomena in a bounded plasma by finite-sized, wide $k_{z}$-spectrum sources. In

*This work was supported by the National Science Foundation under Grant No. GK-40725. 
addition, electron temperatures and densities are measured at pulsed $R F$ power levels of up to $200 \mathrm{~W}$ in order to determine the effect of the excited lower-hybrid waves on electron heating and plasma confinement.

In Section 2 of this paper the experimental device is discussed and methods of measurement are described. In Section 3 the $R F$ potential measurements are presented and compared with theory. In Section 4 measurements of the electron temperature and density are considered. Results are summarized and conclusions are presented in Section 6.

\section{EXPERIMENTAL SETUP}

In order to confirm the theoretical predictions for lower-hybrid waves, the $R F$ electrostatic potential produced by a finite-length split-cylinder source is measured in the plasma with movable probes. These measurements are carried out at $R F$ power levels of less than $100 \mathrm{~mW}$ in $\mathrm{CW}$ operation to ensure that the wave excitation and propagation are linear. At higher power levels, plasma density and electron heating are observed by using probes to measure density and temperature as functions of time in a pulsed- $R F$ mode of operation.

The plasma is generated by a Lisitano coil (LisitaNo, 1965) in a background pressure range of $3 \times 10^{-4}-9 \times 10^{-3}$ Torr and a magnetic field of $1 \mathrm{kG}$ as shown in Fig. 1. The gases A, He, H, D and Ne are used. A pair of split-cylinder plates are excited in the frequency range $2-35 \mathrm{MHz}$. The plates may be driven in phase or out of phase. The $R F$ probes in this experiment are alumina-coated triaxial cylindrical probes composed of a $0.025 \mathrm{~mm}$ center conductor, a floating inner shield and a grounded outer shield. To reduce the effect of the probe capacitance, the inner shield is driven in phase with the signal by an emitter follower circuit. The complete $R F$ detection system is shown in Fig. 2 . These probes may also be used as Langmuir probes that, in the parameter range of the experiment, operate in the orbital-motion-limited regime. A $35-\mathrm{GHz}$ Fabry-Perot open resonator is used as an independent method of checking the probe density measurement. The

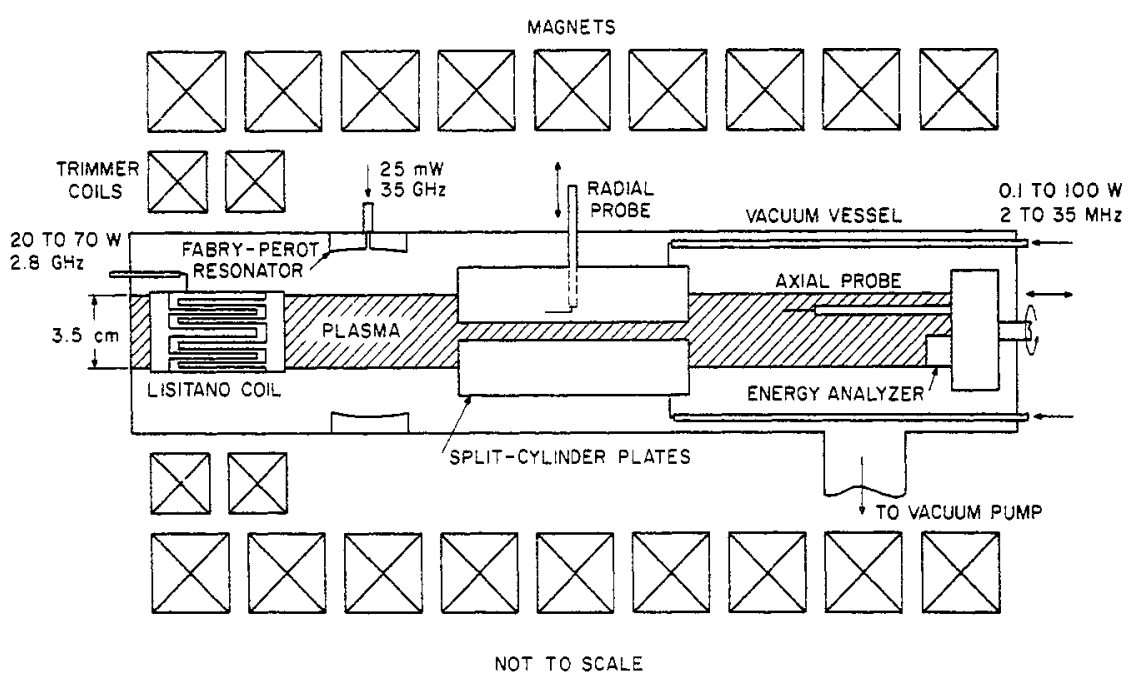

FiG. 1.-Experimental system. The total plasma length is $1.42 \mathrm{~m}$. 


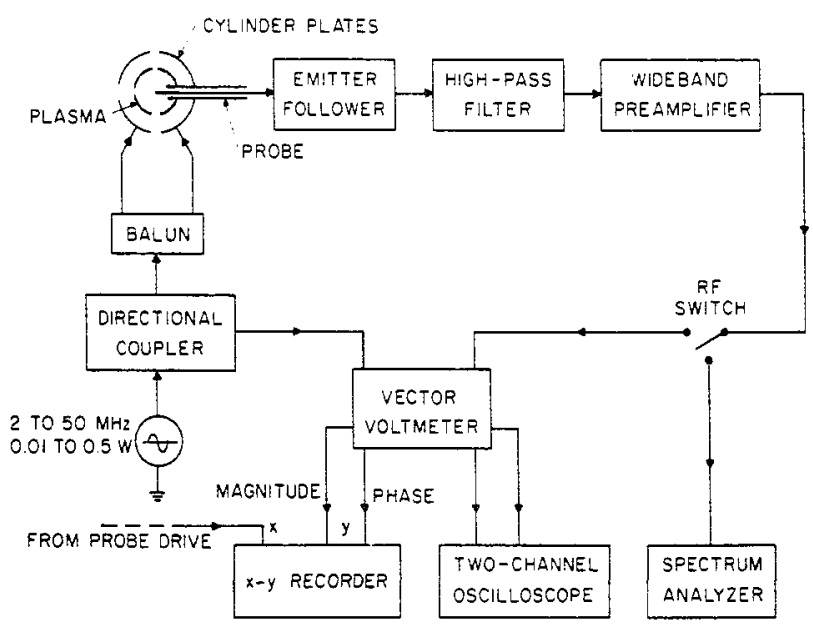

FIG. 2.-RF detection system for CW operation.

measured ion-saturation current radial profile is used in a computer program to extract the density from the resonator frequency-shift measurement. Better than $15 \%$ agreement is found between the two methods over the operating range of the experiment. In pulsed operation, Langmuir-probe measurements are made with $1-\mu$ s time resolution by a sampling technique.

The Lisitano coil is run with $10-70 \mathrm{~W}$ of $\mathrm{CW}$ power at $2800 \mathrm{MHz}$. A pair of small opposing magnetic-field coils is placed outside the vacuum vessel and directly over the Lisitano coil to obtain fine control of the local magnetic-field gradient in the vicinity of the Lisitano coil. The maximum change from the average field value is approximately $20 \%$. This permits plasma generation over a wide range of parameters and gases. A 40:1 range of ion mass is used to check the mass dependence of the lower-hybrid resonance. Except for the small ripple caused by the trimmer coil, the axial magnetic field is uniform to within a few percent.

In pulsed operation the microwave power fed to the Lisitano coil is applied continuously and the $R F$ power applied to the excitation plates is pulsed on for $0.1-1 \mathrm{~ms}$. All probe and analyser measurements are made after the $R F$ pulse is off to avoid $R F$ interference.

The general characteristics and parameters of the steady-state Lisitano-coil plasma are very similar to those of a plasma described by OKTAY and ROBINSON (1973).

\section{WAVE MEASUREMENTS}

Measurement of lower-hybrid waves in this experiment consists of identifying and tracking the resonance-cone surfaces emanating from the split-cylinder source. Measurements of $R F$ potential magnitude and phase were made at the source midplane and several other axial positions as function of radius.

Results of measurements at the source midplane in a neon plasma excited with out-of-phase drive are shown in Fig. 3. As shown in Fig. 3(a), the $R F$ potential magnitude exhibits a peak (labeled "resonance cone") followed by a minimum at 


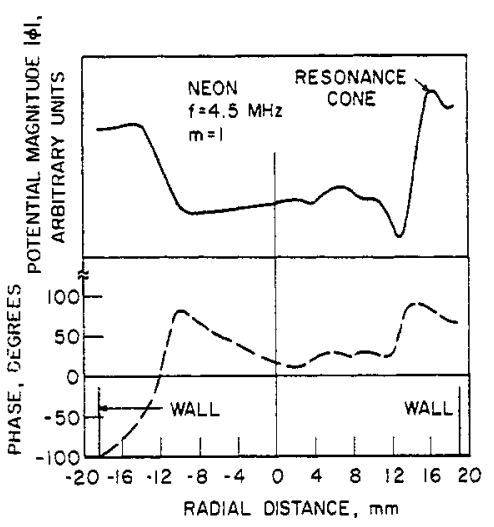

(a)

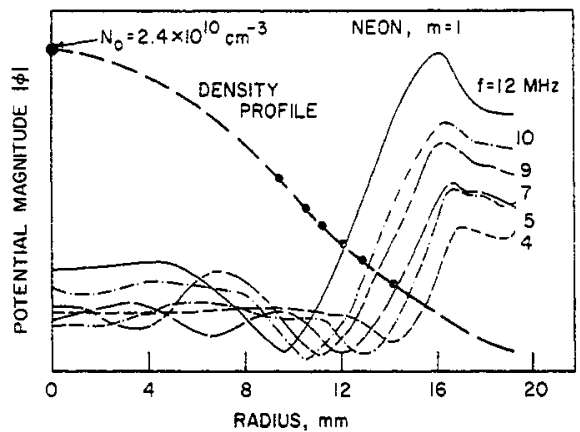

(b)

FIG. 3.-(a) RF electrostatic potential measured across the plasma cross section at midplane of the split-cylinder plates. (b) Radial potential profiles at the midplane for a series of driving frequencies.

slightly smaller radius. The phase changes rapidly with radius with the maximum rate-of-change occurring at the potential minimum. Figure $3(\mathrm{~b})$ shows the effect of varying frequency. As frequency increases the potential minimum moves toward higher density as indicated by the dots on the density-profile curve. The minimum is usually better defined than the potential peak and is used to determine the density dependence of the resonance-cone surface.

In Fig. 4 the density at the $R F$ potential minimum position is plotted as a function of the driving frequency. The experimental density profile of Fig. 3(b) is used but the absolute value of density is uniformly decreased by $20 \%$ from the Langmuir-probe value to match the data points to the theoretical curve at low densities. The $20 \%$ reduction in density is required because the resonance-cone peak always occurs on the low-density side of the minimum.

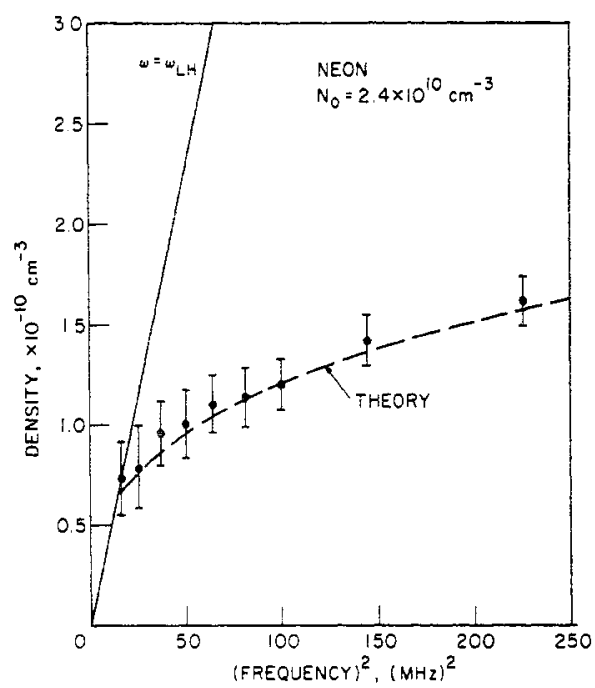

FIG. 4.-Density at the resonance-cone surface at the midplane as a function of driving frequency. Dashed line represents the frequency dependence of the resonance cone density predicted by WKB trajectories. Solid line represents the lower-hybrid resonance condition $\omega=\omega_{L H}=\omega_{\text {pi }}$. 
Similar plots of ineasured resonance-cone densities for four ion species are given in Fig. 5 as functions of frequency, which is normalized by the ratio $\left(m_{i} / m_{\mathrm{He}}\right)^{1 / 2}$. This normalization removes the ion-mass dependence of the ionplasma frequency so that the relationship between the resonant-cone density and the frequency is independent of ion mass for a given density profile. The results of Fig. 5 show qualitative agreement with theory over a $40: 1$ range of mass. No corrections were made for density or variations of density profiles in Fig. 5.

The dependence of resonance-cone density on frequency is a rather special case when measured at the source midplane as will be discussed in Section 5 . It should be noticed in Fig. 4 that the frequency falls well below the lower-hybrid resonance frequency $\omega_{L H} \simeq \omega_{p i}$, where $\omega_{p i}$ is the ion plasma frequency. This effect was first noted by Hooke and BerNABeI (1972).

Measurements of the $R F$ potential at a series of axial positions all of which were outside of the source are shown in Fig. 6 . In the case shown the frequency exceeded the maximum lower-hybrid frequency in the plasma and thus no resonance layer exists in the plasma. The plasma response is characterized by a prominent $R F$ potential maximum that generally follows the characteristic curves defining the resonance-cone surfaces. A definite reflection of the maximum from the column axis is observed along with a gradual leveling of the $R F$ radial profile because of collisional damping. The results of similar measurements for the case when the lower-hybrid resonance layer occurs within the plasma are shown in Fig. 7. In this case the resonance layer occurs at a radius of approximately $5 \mathrm{~mm}$ and the maxima in the response are roughly asymptotic to this layer. The radial variation of potential magnitude for various frequencies at axial locations other than the midplane show results similar to those of Fig. 3 . When the plates are

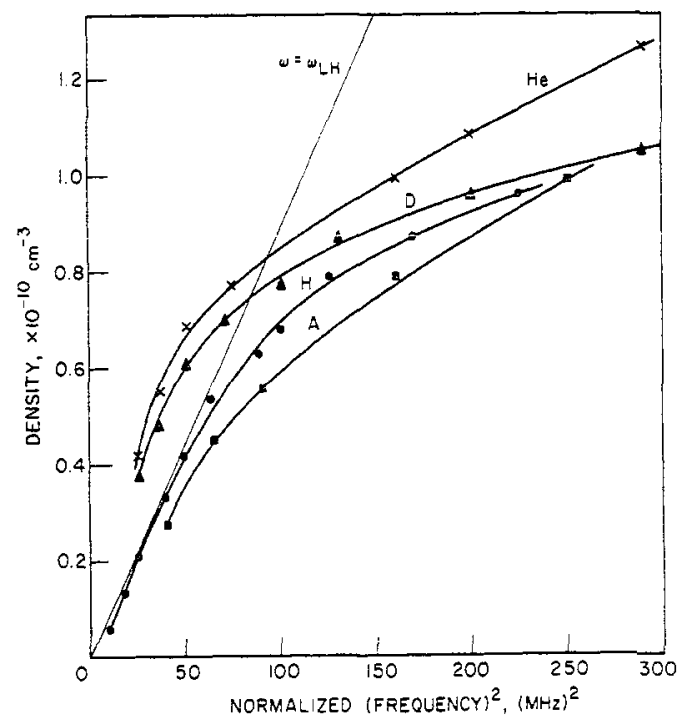

FIG. 5.-Resonance-cone density at the midplane as a function of normalized driving frequency for several gases. The measured frequency is scaled by $\sqrt{ } m_{i} / m_{\mathrm{He}}$ to remove the ion dependence of the lower-hybrid resonance. 


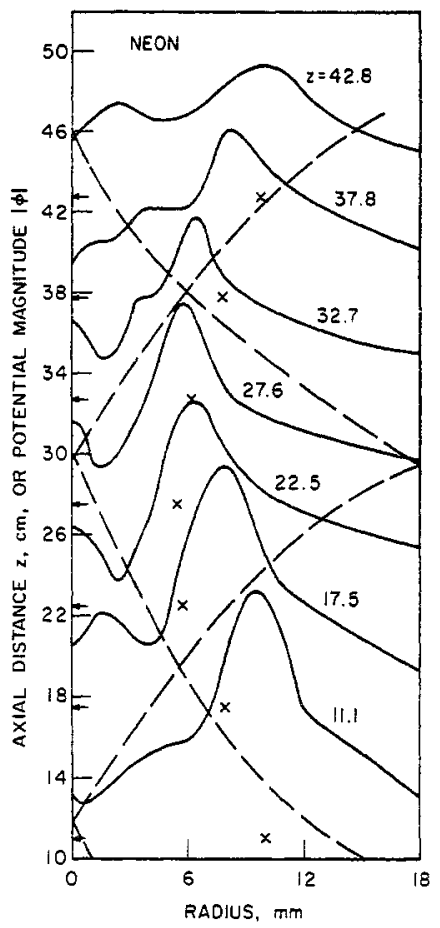

FIG. 6.-Radial potential profiles for the case $\omega>\omega_{L H}$ at a series of axial positions outside the plates. Plate length is $18 \mathrm{~cm}$. Dashed lines represent the WKB resonant-cone trajectories. X's represent loci of the maxima. The origins for each curve are indicated by arrows at the corresponding axial positions on the ordinate. $\left(f=10 \mathrm{MHz}, N_{0}=4.1 \times\right.$ $10^{9} \mathrm{cis}^{-3}, B_{0}=1.3 \mathrm{kG}$ and $m=1$ ).

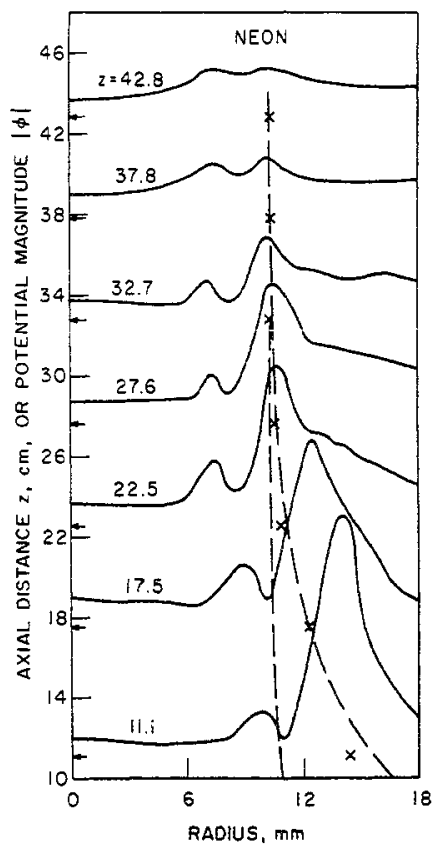

FIG. 7.-Radial potential profiles for the case $\omega<\omega_{L H}$ at a series of axial positions outside the plates. Plate length is $18 \mathrm{~cm}$. Dashed lines represent the WKB resonant-cone trajectories. X's represent loci of the maxima. $\left(f=5.8 \mathrm{MHz}, N_{0}=2.0 \times 10^{10} \mathrm{~cm}^{-3}, B_{0}=\right.$ $1.3 \mathrm{kG}$ and $m=1)$. 
driven in phase and as the driving frequency is increased, the maximum moves toward the column axis and merges into a single central peak. The same qualitative resonance-cone behavior is observed for sources of various lengths from $18-70 \mathrm{~cm}$.

\section{PARTICLE MEASUREMENTS}

As discussed in Section 2, the electron temperature is measured with Langmuir probes. The measurements were made in $\mathrm{He}$ and $\mathrm{Ne}$ plasmas after a 1-ms $R F$ pulse as the plasma decays back to the steady-state Lisitano-coil-produced plasma. The background plasma has many of the characteristics of the argon plasma studied by OKTAY and ROBINSON (1973). The electron-temperature radial profile has a peak of approximately $2.3 \mathrm{eV}$ and the plasma potential has a relatively sharp jump of approximately $1.5 \mathrm{eV}$ near the point of maximum density gradient. The average electron temperature over the column cross section is 1.0 to $1.5 \mathrm{eV}$. The peak ion temperature of the background plasma is found to range from 0.2 to $0.8 \mathrm{eV}$ for microwave power inputs of $10-70 \mathrm{~W}$ in agreement with Oktay and Robinson. The density profile of the background plasma is essentially Gaussian and varies by less than $15 \%$ over the $150-\mathrm{cm}$ plasma length.

The results of the electron-temperature analysis of the Langmuir probe curves for three axial positions are shown in Fig. 8. These results are obtained with a time resolution of $1.0 \mu \mathrm{s}$ but depend on pulse-to-pulse reproducibility. The electron temperature at the center and near the plasma edge is highest just after the $R F$ pulse and decays back to steady-state conditions in 200-300 $\mu \mathrm{s}$. The temperature at the edge is slightly higher than the temperature at the center for

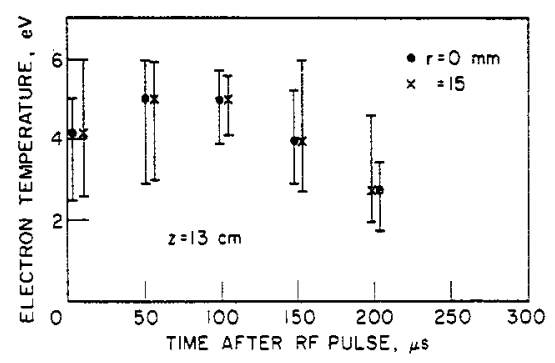

(a)

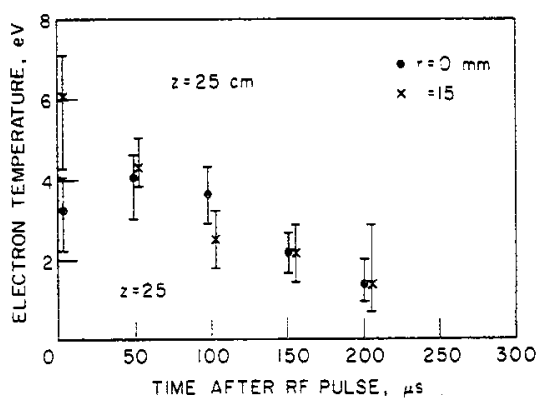

(b)

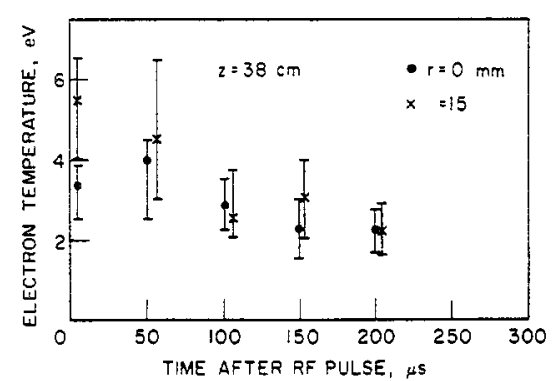

(c)

FIG. 8.-Electron temperature in the afterglow of the $R F$ pulse at the column center and near the edge for three axial positions. (Neon, $B_{0}=1.2 \mathrm{kG}, P_{\text {in }}=10 \mathrm{~W}, f=$ 14.3 $\mathrm{MHz}$ and pulse length $=1 \mathrm{~ms}$ ). 
$50 \mu \mathrm{s}$ after the pulse. At later times the axis and edge temperatures are equal. The estimated electron temperature increase immediately after the $R F$ pulse is approximately $5 \mathrm{eV}$ for an absorbed $R F$ power of $0.3 \mathrm{~W}$. It was found that $R F$ absorbed power increased as the square of the source-electrode voltage which is consistent with a linear, resistive power absorption process.

The density variation during and after the $R F$ pulse was measured with Langmuir probes and the Fabry-Perot resonator. Since the probe current in the ion-saturation regime is proportional to density, the time variation of density can be observed as shown in Fig. 9. A sharp decrease in density to approximately one third the steady-state value occurs during the $R F$ pulse. A similar decrease is found with the Fabry-Perot resonator. As shown in Fig. 10(a) the radial density profile is diminished predominately near the column center. The density as measured by the interferometer during the $R F$ pulse decreases nearly linearly with increasing incident $R F$ power until approximately $70 \mathrm{~W}$ is reached as shown by Fig. 10(b). At that point no further decrease in density occurs for higher incident power, but the plasma volume expands and the plasma is sustained by the pulsed $R F$ alone.

Figure 9 also shows a low-frequency oscillation in the ion-saturation curve after the $R F$ pulse is turned off. This oscillation, which in Fig. 9 is at $3 \mathrm{kHz}$, is found in a narrow range of incident power and background pressure. It has been observed previously and identified as an ion acoustic wave (Raimbault and SHOHET, 1975).

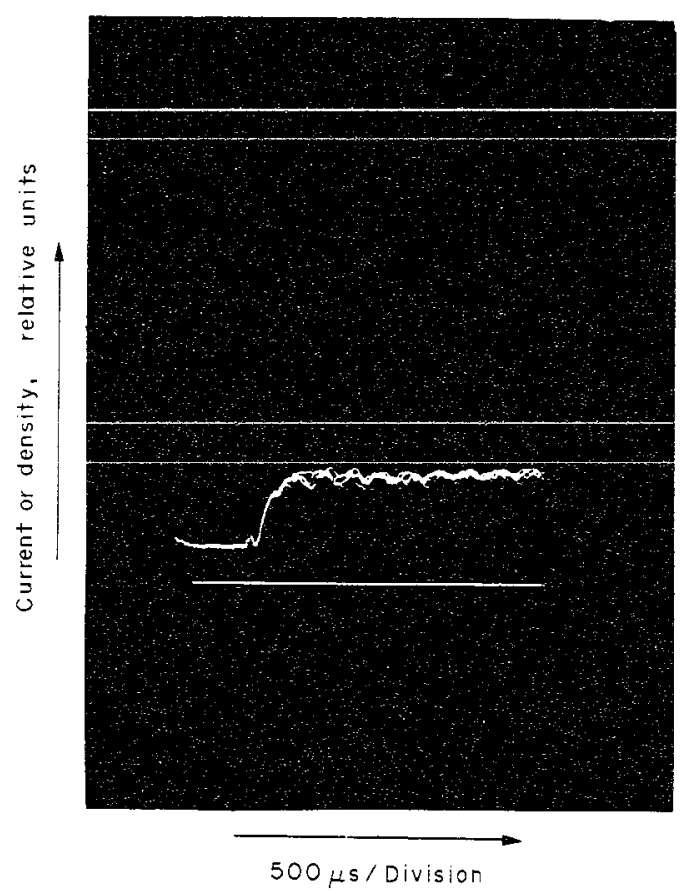

FIG. 9.-Ion saturation current as a function of time during the $R F$ pulse. (Neon, $P_{\text {in }}=50 \mathrm{~W}$ and pulse length $=1 \mathrm{~ms}$ ). 


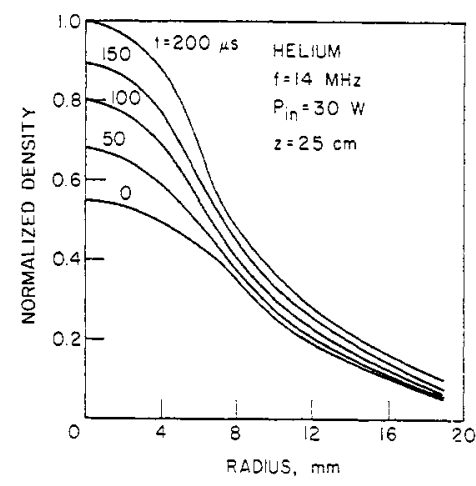

(0)

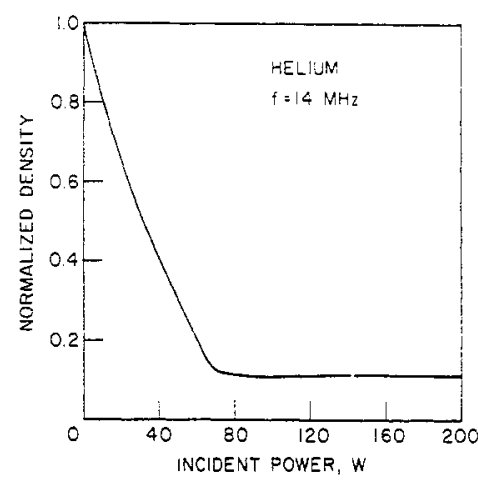

(b)

FIG. 10.-(a) Density profile for a series of times in the afterglow and (b) normalized density during the $R F$ puise as a function of the incident power.

\section{DISCUSSION}

Properties of the resonance cones are described by their WKB trajectories in the manner given by BELLAN and PORKOLAB (1974). Their results are modified for cylindrical geometry and the experimentally-determined density profile is used to calculate the WKB trajectories from the equation

$$
z \pm \int^{r}\left(-\frac{P\left(r^{\prime}\right)}{S\left(r^{\prime}\right)}\right)^{1 / 2} \mathrm{~d} r^{\prime}=\text { constant }
$$

where $P(r)$ and $S(r)$ are the radius-dependent cold-plasma dielectric tensor components. The trajectories emanate from the ends of the source and travel axialiy and radiaily in accordance with (1). In the source midplane the WKB trajectories from opposite ends of the source intersect at a single radial position. The intersection point is on the low-density side of the resonance layer and its radius is used with the experimental density profile to determine the theoretical curve of Fig. 4. Because of the long axial distances required for the trajectories to reach the lower-hybrid resonance layer, the frequency dependence of the resonance-cone density at the midplane departs significantly from the lowerhybrid resonance condition. The amount of departure of the density from the resonance condition depends on the density profile but for plasmas with the same profile but different ion masses, the departure scales as $m_{i}^{1 / 2}$. This behavior is confirmed within experimental error as shown by Fig. 5 .

The trajectories are shown in the $r-z$ plane by the dashed curves in Figs 6 and 7. In the low-density case of Fig. 6 the trajectories appear to reflect from the column axis, while in the high-density case in Fig. 7 they are asymptotic to the resonance layer. In Fig. 6 the RF potential peaks are marked by an $x$ for each axial position. These points tend to follow the WKB trajectories and the resonance-cone surfaces appear to reflect. For the case of Fig. 7 the large $R F$ potential peak marked by $x$ 's tends to follow the WKB trajectories. The two peaks in the figure correspond to resonance cones from opposite ends of the source; the higher peak originates from the near end and the lower peak originates from the far end, being reduced significantiy by damping over the longer distance. 
Differences between the radial locations of the resonance cones and the WKB trajectories as shown in Figs. 6 and 7 are within the experimental accuracy of the density profile measurements that are used to calculate the trajectories. It should be noted that detailed computer calculations of the plasma response gives excellent agreement with the entire $R F$ potential radial profile as well as with the WKB trajectories (COLESTOCK and GETTY, 1976). These calculations are based on the superposition of bounded-plasma eigenmodes of a wide range of closely spaced values of $k_{z}$. The eigenmodes are calculated for inhomogeneous plasma and are similar to the well-known plasma waveguide modes for the homogeneous plasma (TRIVELPIECE and GOULD, 1959). Similar superposition solutions have been presented recently by LAZZARO (1975).

The electron temperatures measured by Langmuir probes do not exhibit a well-defined spatial dependence as can be seen in Fig. 8. A typical temperature increase of $5 \mathrm{eV}$ is observed, which is similar to the increase measured by Raimbault and Shohet under comparable conditions. However, they observed electron heating only on the low-density side of the lower-hybrid resonance layer. In this experiment the density is sufficiently low so that no resonance layer occurs and electron heating penetrates to the center. The equilibrium electron temperature may also be found from the ion-acoustic wave seen in the pulse decay in Fig. 9. The oscillation frequency is given by

$$
\nu=\frac{1}{L}\left(\frac{k T_{e}}{m_{i}}\right)^{1 / 2},
$$

where $L$ is the plasma length. For the measured equilibrium temperature (2) yields an oscillation frequency of $2 \mathrm{kHz}$ which is near the measured value. The plasma decay time observed in Fig. 9 is consistent with a loss rate given by the ion streaming time $L / v_{t i}$.

\section{SUMMARY AND CONCLUSIONS}

The principal result of this study is a confirmation of the resonance-cones in the plasma response to excitation by a finite-length source. The resonance-cone surfaces are shown to have the predicted frequency, density and ion-mass dependence according to linear, electrostatic wave theory in a bounded plasma. For frequencies near the lower-hybrid frequency, the resonance cone surfaces extend over long axial distances from the source before either reaching the resonance layer or reflecting from the column axis. The presence of damping in the plasma causes the resonance cones to disappear with axial distance. At power levels above a few watts electron heating is observed and is accompanied by increased plasma end losses from the device. It is concluded that the applied $R F$ fields heat the electrons by several electron volts.

\section{REFERENCES}

1. Bellan P. M. and Prokolab M. (1974) Physics Fluids 17, 1592.

2. Bellan P. M. and Porkolab M. (1975) Phys. Rev. Lett. 34, 124.

3. Briggs R. J. and Parker R. R. (1972) Phys. Rev. Lett. 29, 852.

4. Colestock P. L. and GeTry W. D. (1974) Proc. 2nd Topical Conf. on RF Plasma Heating, Paper B-3. Texas Tech. Univ., Lubbock, Texas.

5. Colestock P. L. and GetTy W. D. (1976) Physics Fluids, 19, 1229. 
6. Hooke W. M. and Bernabei S. (1972) Phys. Rev. Lett. 28, 407.

7. Lazzaro E. (1975) Plasma Phys. 17, 1033.

8. Lisitano G. (1965) Proc. 7th International Conf. on Ionization Phenomena in Gases, p. 464. Belgrade.

9. MoResco M. (1973) Phys. Lett. 45A, 65.

10. Oktay E. and Robinson J. E. (1973) Plasma Phys. 15, 227.

11. Raimbault P. A. and Shohet J. L. (1975) Plasma Phys. 17, 327.

12. Simonutti M. D. and PArker R. R. (1974) Proc. 2nd Topical Conf. on RF Plasma Heating, Paper B-2. Texas Tech. Univ. Lubbock, Texas.

13. Stenzel R. and Gekelman W. (1975) Phys. Rev. A11, 2057.

14. Trivelpiece A. W. and Gould R. W. (1959) J. appl. Phys. 30, 1784. 